\title{
Sentido de vida e saúde mental durante o tratamento de câncer
}

\author{
Randolfo Santos Junior* \\ Caroline Santos de Simone** \\ Gabriela Volpe Pinhabel*** \\ Valeria Aparecida Teixeira Galhardo*** \\ Aline Carniel***
}

\begin{abstract}
Resumo
Câncer é uma doença crônica de alta prevalência e que impõe desafios de ajustamento ao paciente e seus familiares. Os objetivos deste estudo foram avaliar a presença de sentido de vida em pacientes com câncer e sua correlação com outras variáveis psicossociais. Material e Método: Participaram 91 pacientes em tratamento oncológico há no mínimo três meses. Instrumentos: Questionário Sóciodemográfico; Escala Hospitalar de Ansiedade e Depressão; Escala de Esperança de Herth; Mini-Mac; Escala de Apoio Social. Resultados: Observa-se uma correlação positiva entre os indicadores de sentido de vida e os indicadores de esperança, apoio social, tempo de diagnóstico e rede de apoio. E uma correlação negativa com os indicadores de depressão, preocupação ansiogênica e desamparo. Conclusão: Há evidências de que a presença de sentido associada a outras variáveis é um possível indicador de proteção à saúde mental dos pacientes em tratamento de câncer.
\end{abstract}

Palavras-chave: Câncer; Psicologia da saúde; Ajustamento emocional;

\begin{abstract}
Cancer is a chronic disease of high prevalence and that imposes adjustment challenges to the patient and their relatives. The objectives of this study were to evaluate the presence of life meaning in patients with cancer and its correlation with other psychosocial variables. Material and Method: 91 patients undergoing cancer treatment for at least three months. Instruments: Socio-demographic questionnaire; Hospital Anxiety and Depression Scale; Herth's Scale of Hope; Mini-Mac; Social Support Scale. Results: There is a positive correlation between the indicators of life expectancy and the indicators of hope, social support, diagnosis time and support network. And a negative correlation with the indicators of depression, anxiogenic worry and helplessness. Conclusion: There is evidence that the presence of sense associated with other variables is a possible indicator of the protection of the mental health of patients in cancer treatment.
\end{abstract}

Keywords: Cancer; Psychology; Psychological Adjustment

\footnotetext{
* Psicólogo pela UFU, Doutor pela FAMERP e Chefe do Serviço de Psicologia do Hospital de Base. Docente do PPG da FAMERP.

** Psicóloga pela UNIPSJRP e Especialista em Psicologia da Saúde pela FMSJRP.

***Psicóloga e Especialista pela FMSJRP.
} 


\section{Introdução}

Uma doença crônica pode se constituir como um fator de risco significativo à saúde mental em virtude da exposição prolongada a estressores e incertezas relacionadas ao tratamento, aos prejuízos na qualidade de vida, modificações na autoimagem, presença de sintomas, efeitos adversos de medicações e afastamento de papeis sociais. (Barbosa \& Santos, 2012; Snoerck, Bremmer \& Hermanns, 2015; Sharafkhaneh, Yohannes, Hanania, \& Kunik, 2017; Yoong, Mooppil, Khoo, Newman, Lee, Kang \& Griva, 2017; Dua, Das, Kumar, Kumar, Mishra, \& Sharma, 2018).

Porém, a vivencia de uma doença crônica não é por si um determinante para o sofrimento emocional significativo e persistente. O impacto da doença será influenciado principalmente pela forma como o sujeito interpreta a situação de adoecimento, como identifica e utiliza seus recursos pessoais de enfrentamento e suas fontes de apoio ambiental e espiritual, que podem em certo sentido verterem-se em fatores de proteção, amortecendo o impacto dos estressores e favorecendo um ajustamento saudável as transformações advindas do adoecimento. (Vickberg, Bovbjerg, DuHamel, Currie, \& Redd, 2000; Boscaglia \& Clarke, 2007; Casale, Wild, Cluver \& Kuo, 2015; Vanderlan, Kurtz, Sladky, Asvat \& Deshields, 2016).

O processo de ajustamento a uma doença crônica passa inevitavelmente na maioria dos casos por uma reestruturação profunda de expectativas e crenças suscitando questões inquietantes relacionadas ao seu propósito existencial e a o sentido da vida. (Sherman \& Simonton, 2012; Dezutter, Casalin, Wachholtz, Luyckx, Hekking, \& Vandewiele, 2013; Bronk, 2014).

Frankl (1984) em suas obras seminais sobre os aspectos protetores da presença de sentido em circunstancias adversas, propõe que se por um lado o ser humano vivencia em sua trajetória situações de crise que expõe a fragilidade das suas crenças de controle por outro lado, tem a possibilidade de ressignificar sua existência e estruturar novos sentidos para sua vida. Tal premissa tem sido investigadas em estudos recentes como o de Bronk (2014) que aponta a presença de sentido de vida como uma variável ligada a maior bem-estar, qualidade de vida e emoções positivas em situações de crise e adoecimento.

O presente trabalho tem como objetivo geral investigar o sentido de vida dos pacientes em tratamento de câncer. Ou seja, sentido de vida como uma variável de proteção que pode ajudar o paciente no ajustamento psicológico.

\section{Materiais e método}

\section{Participantes}

Participaram da pesquisa 91 pacientes em tratamento oncológico no Hospital de Base (HB), Instituto do Câncer (ICA) e Unidade de Quimioterapia, adultos (idade superior a 18 anos), de ambos os sexos, em tratamento há pelo menos 3 (três) meses - período mínimo para ajustamento psicossocial. Foram excluídos participantes com dificuldades de audição e ou/ fala, ou com déficit cognitivo apontado em prontuário.

\section{Instrumentos}

Para o processo de coleta de dados os participantes responderam aos seguintes instrumentos:

1) Questionário inicial de dados Sóciodemográfico. Instrumento de autoria dos pesquisadores, foi construído com o objetivo de caracterizar os participantes quanto aos aspectos demográficos e clínicos, que investiga: na primeira parte - (através de uma pesquisa ao prontuário do paciente): diagnóstico; tempo de diagnóstico, presença de metástase, na segunda parte, (indagando ao próprio paciente): idade, sexo, endereço, escolaridade, estado civil, quantidade de filhos, raça, atividade profissional e, religião (Apêndice 1);

2) Escala de Ansiedade e Depressão Hospitalar - HAD (Escala para avaliar ansiedade e depressão em adultos). Suas principais características são: sintomas vegetativos que podem aparecer em doenças físicas foram evitados; os conceitos de depressão e ansiedade encontram-se separados, o conceito de depressão encontra-se centrado na noção de anedonia; inclina-se a detectar graus leves de transtornos afetivos em ambientes não psiquiátricos; é curta, podendo ser rapidamente aplicada e preenchida; ao paciente solicita-se e é recomendado que responda baseando-se em como se sentiu durante a última semana. Sendo 14 questões de múltipla escolha e duas sub-escalas (ansiedade e depressão) com sete itens cada, (ponto de corte 8 ). Serão adotados os pontos de corte apontados por Zigmond e Snaith (1983), recomendados para ambas as situações: de 0-7 pontos: improvável, 8-11 pontos: possível (questionável ou duvidoso) e 12-21: provável. As análises serão feitas a partir das normas propostas de acordo com a validação elaborada por Botega, Bio, Zomignani, Garcia Jr., \& Pereira, (1995).

3) Escala de esperança de Herth : A escala originou-se do instrumento americano Hert Hope Index e possibilitou a existência de um instrumento específico para 
mensuração da esperança, antes inexistente no Brasil, chamada de Escala de Esperança de Herth - EEH (Hert, 1991). É uma escala de autorrelato de origem americana, que quantifica a esperança de vida. Esta escala possui 12 afirmativas e a graduação dos itens segue a escala do tipo Likert de quatro pontos, variando de "concordo completamente" a "discordo completa mente" onde 1 indica "discordo completamente" e 4 corresponde a "concordo completamente". Os itens 3 e 6 apresentam escores invertidos. O escore total varia de 12 a 48 e quanto maior o escore, mais alto o nível de esperança de vida. É uma escala considerada breve e de fácil compreensão e demonstrou prioridades psicométricas adequadas.

4) Escala de Apoio Social: Composto por 19 itens compreendendo cinco dimensões funcionais de apoio social: material, afetiva, emocional, interação social positiva e informação (Griep, Chor, Faerstein, Werneck \& Lopes, 2005).

5) Questionário de Sentido de Vida: Formado por 10 questões avaliadas em uma escala de 7 pontos variando de $1=$ totalmente falso a $7=$ a Totalmente verdadeiro. Apresenta dois fatores: Presença de sentido e busca de sentido (Aquino, Veloso, Aguiar, Serafim, Pontes, Pereira \& Fernandes, 2015).

6) Escala de Ajustamento Mental para o câncer (MINI-MAC): Trata-se de um instrumento constituído por 2 (dois) fatores:- Fator 1 - Preocupação Ansiógena: apresenta 8 (oito) afirmações como, por exemplo, "Estou preocupada com minha doença" e "Sinto-me agoniada com minha doença"; Fator 2 - Desamparo/Desesperança: apresenta 5 (cinco) afirmações, entre elas, "Sinto que esta doença está ale, das minhas forças" e "Não consigo lutar contra minha doença".

Nos dois fatores que constituem a escala, as respostas para as afirmações são apresentadas desde "Nunca" até "Sempre" em uma escala do tipo Likert de 4 pontos, onde conforme maiores os valores apresentados piores as condições de Preocupação Ansiógena e Desamparo/ Desesperança (Gandini, Martins, \& Pedrosa, 2008).

\section{Procedimentos}

Trata-se de uma pesquisa transversal, descritiva-exploratória. Os pacientes que estiveram em tratamento oncológico no Instituto do Câncer - ICA e Hospital de Base de São José do Rio Preto (HB), foram convidados durante hospitalização e nas sessões de quimioterapia para participarem da avaliação psicológica, foram orientados quanto ao tema e objetivos da pesquisa; sua não obrigatoriedade de participação; anonimato e aspectos éticos; interrupção por parte do paciente se o mesmo julgasse necessário; foi apresentado e realizado leitura do termo de Consentimento Livre e Esclarecido (Modelo em acordo com a Resolução no 466/12 - Conselho Nacional de Saúde), contendo dados de identificação do pesquisador e dos participantes, objetivos e procedimentos da pesquisa - Após assinatura do Termo foi realizada aplicação dos questionários e escalas individualmente. Foi disponibilizado suporte emocional ao paciente.

A coleta de dados foi iniciada após a aprovação do projeto pelo Comitê de Ética em Pesquisa da Faculdade de Medicina de São José do Rio Preto - SP (FAMERP). Foi realizado no início dos atendimentos, os esclarecimentos necessários sobre os objetivos e utilização acadêmica e científica dos dados. Os participantes que concordaram e assinaram o Termo de Consentimento Livre e Esclarecido.

\section{Análise de dados}

Para a análise dos dados foram utilizados: estatística descritiva, apresentação dos dados clínicos e sociodemográficos, o Teste de Mann Whitney para comparação de médias entre grupos de participantes e o Teste de Spearman para a análise de correlação entre as variáveis com nível de significância de $\mathrm{p}<0.005$.

\section{Resultados}

Para a realização da pesquisa foram entrevistados 91 pacientes. Quanto às suas características, observou-se o predomínio de mulheres $(56,04 \%)$, a idade média dos participantes foi de 54,5 ( \pm 13.443) anos e variou de 21 a 77 anos. Em relação ao estado civil, a maioria dos participantes eram casados (63,74\%), quanto à escolaridade, observou-se a presença de uma baixa escolaridade com a maioria $(41.75 \%)$, cursando somente o primeiro grau incompleto. No tocante a religiosidade $(58.24 \%)$ eram católicos e a frequência com que vão a alguma celebração religiosa, a maioria relatou ir de duas ou mais vezes na semana $(24.40 \%)$

Em relação aos dados clínicos, foi observado a predominância de participantes com diagnóstico primário de tumores de mama $(23,07 \%)$, seguido de Colorretal $(19,80 \%)$, Pulmão (9,89\%), Cabeça e pescoço (6,59\%), Linfomas (6,59\%) e outros (34,06\%). O tempo de diagnóstico variou de 3 a 12 meses $(58,26 \%)$, de 12 a 24 meses $(19,78 \%)$, de 24 a 36 meses $(10,98 \%)$ e mais de 36 meses em tratamento $(10,98 \%)$.

Dessa amostra, 20,9\% dos participantes apresentaram indicadores de depressão, 25,3\% apresentaram indicadores de ansiedade. Quanto aos indicadores de esperança, os escores variaram de 26 a 50 pontos e a média da amostra foi de $(40,9 ; \pm 5.140)$. 
Tabela 1 - Dados sociodemográficos dos participantes

Variáveis

\section{Sexo}

Masculino

Feminino

\section{Escolaridade}

Não frequentou a escola

Ensino Fundamental Incompleto

Ensino Fundamental Completo

Ensino Médio Incompleto

Ensino Médio Completo

Universitário Incompleto

Universitário Completo

\section{Estado civil}

Casado

Divorciado

Solteiro

Viúvo

\section{Orientação Religiosa}

Não possuo religião/ Não acredito em Deus

Acredito em Deus, mas não sigo nenhuma religião

Católico

Evangélico

Espírita
$41 \quad 45,06$

$50 \quad 54,94$

$2 \quad 2,19$

$38 \quad 41,79$

$9 \quad 9,89$

$\begin{array}{ll}8 & 8.79\end{array}$

$19 \quad 20,87$

$1 \quad 1,09$

$14 \quad 15,38$

$58 \quad 63,74$

$11 \quad 12,09$

$14 \quad 15,38$

$8 \quad 8,79$

$0 \quad 0$

$4 \quad 4,39$

$53 \quad 58,24$

$27 \quad 29,68$

\begin{tabular}{l}
7 \\
\hline
\end{tabular} 
Observa-se uma correlação positiva entre os indicadores de sentido de vida e os indicadores de esperança, apoio social, tempo de diagnóstico e rede de apoio. E uma correlação negativa com os indicadores de depressão, preocupação ansiogênica e desamparo.

Tabela 2 -Correlação entre sentido de vida e outras variáveis Coeficiente de Spearman.

$\begin{array}{lcc}\text { Variáveis } & \text { Sentido de vida } & \boldsymbol{P} \\ \text { Ansiedade } & \mathrm{r}=-0,3222 & 0,0043^{*} \\ \text { Depressão } & \mathrm{r}=-0,3162 & 0,0051^{*} \\ \text { Tempo de diagnóstico } & \mathrm{r}=0,2505 & 0,0280^{*} \\ \text { Esperança } & \mathrm{r}=0,2523 & 0,0269^{*} \\ \text { Apoio Social } & \mathrm{r}=0,2776 & 0,0077^{*} \\ \text { Mini Mac - desamparo } & \mathrm{r}=-0,3354 & 0,0029^{*} \\ \text { Idade } & & \mathrm{r}=0,1599\end{array}$

\section{Discussão}

Neste estudo, a presença de sentido de vida mostrou-se associada a maior bem-estar emocional durante o tratamento de câncer caracterizado por menos indicadores de ansiedade, depressão e desesperança entre os participantes com indicadores mais elevados de presença de sentido.

Estudos tem mostrado que a presença de sentido de vida pode funcionar como um fator de proteção para estressores da vida diária (Park \& Baumeister, 2017) estabelecendo uma associação positiva com maiores indicadores de bem estar (Yalçin \& Malkoç, 2014; Datu, 2015), enfrentamento ativo focado no problema (Cho, Lee, Lee, Bae \& Jeong, 2014), satisfação com a vida, auto estima e ajustamento psicológico (Dezutter et al; 2013).

Maior presença de sentido para a vida também é associado com melhor saúde psicológica. Um estudo que aponta uma correlação negativa entre presença de sentido e depressão, destaca também uma associação significativa entre sentido de vida, saúde mental e interações sociais. Estar envolvido em relacionamentos significativos, e em atividades sociais, políticas, esportivas e culturais está associado a redução da sintomatologia da depressão e, aumento de significado da vida. (Kleftaras \& Psarra, 2012). Entre os participantes desta pesquisa a presença de apoio social significativo mostrou-se fortemente correlacionado com presença de sentido. Em um importante artigo Letborgh, Aranda \& Kissane (2008) ressaltam que as principais diretrizes de cuidado a um paciente com câncer envolvem: promover o alivio do sofrimento físico; reconhecer o sofrimento em todas as suas dimensões; encorajar a busca de sentido e propósito; e fortalecer a conexão com as outras pessoas. Para estes autores, o câncer gera uma experiência de descontinuidade na história de vida e que, ao compartilhar sua história a pessoa poderá então expressar sua dor existencial e assim processar sua percepção da doença e das suas transformações tendo preservado um sentido de pertencimento e de conexão com o outro, o indivíduo teria assim elementos fundamentais para uma ressignificação da vida, e para a manutenção de um senso de propósito.

Um estudo recente concluiu que mesmo em fases avançadas de uma doença crônica o suporte social e o sentido de vida podem contribuir para uma melhor 
percepção da qualidade de vida dos pacientes. Os resultados de uma investigação com 114 pacientes atendidos em serviços de cuidados paliativos apontam uma relação entre a frequência de visitas e o apoio social oferecido por um parente próximo a maior bem-estar e sentido de realização por parte dos pacientes. (Dobríková, Pčolková, AlTurabi \& West Jr, 2015) No mesmo caminho, um outro estudo que buscou explorar a relação entre sentido de vida, apoio social e ideação suicida em pacientes com câncer aponta que o apoio de amigos e familiares foi um fator importante para redução do risco de suicídio e fortalecimento do sentido de vida após o diagnóstico. (Kim \& Lee, 2010).

Ferreira e Guerra (2014), avaliando fatores de adaptação psicológica em pacientes com lesões vertero-modular observaram correlação positiva entre o sentido de vida felicidade e suporte social e correlação negativa entre o sentido de vida e indicadores de depressão influenciando na qualidade de vida e processo de adaptação dos participantes.

Durante as entrevistas para coleta dos dados tornou-se evidente que a vivência de um estressor crônico como uma doença grave ou uma perda significativa pode alterar radicalmente o processo de desenvolvimento de uma pessoa e seu entorno e ser um fator de vulnerabilidade a saúde mental e qualidade de vida, mesmo após a cessação do evento traumático. No entanto, não existe uma determinação absoluta neste processo, pelo contrário, entre os participantes foi observado que um maior tempo de convivência com a doença pode favorecer uma maior presença de sentido para a vida. Frente a perspectiva de superação de uma experiência desafiadora, muitas pessoas relatam uma maior valorização e satisfação com a vida e um maior senso de propósito permeado por uma perspectiva de ajustamento positivo as experiências ocorridas que forma denominadas por Tedeschi e Calhoun (1996) de crescimento pós-traumático.

Tendo em vista que o crescimento pós-traumático é considerado uma resposta adaptativa a um estressor grave e que este processo implica em uma capacidade do indivíduo de elaborar uma reestruturação cognitiva de crenças, expectativas e propósitos de vida, pode-se considerar a importância de aspectos pessoais e ambientais que podem favorecer essa tarefa de ressignificação. Pesquisas apontam que o crescimento pós traumático pode ser favorecido por características pessoais como flexibilidade emocional, saúde mental e presença de sentido (Schroevers, Helgeson, Sanderman, \& Ranchor, 2010; Kashdan \& Kane, 2011; Casellas-Grau, Ochoa, \& Ruini,
2017), enfrentamento religioso positivo, e otimismo (Pratti \& Pietrantoni, 2009); e também por uma percepção de apoio emocional significativo (Zeligman, Varney, Grad \& Huffstead, 2018); e do suporte social como mediador de melhor auto estima e maiores indicadores de esperança (Zhou, Wu \& Zhen, 2018). Estes resultados apontam para a importância da identificação destas variáveis no perfil psicossocial de pacientes com câncer, e principalmente para a necessidade de elaborar intervenções que possam promover a manutenção e o desenvolvimento de fatores de proteção em face dos estressores do tratamento.

Assim como em outros estudos, Não foi observada diferenças na presença de sentido entre os gêneros (Dezutter et al, 2013; Eakman \& Eklund, 2012) e idade dos participantes (Dezutter et al, 2013; Eakman \& Eklund, 2012; Ortiz \& Morales, 2013; Cho, Lee, Lee, Bae \& Jeong, 2014).

A depressão não é necessariamente um desfecho certo para pessoas acometidas por demandas crônicas de saúde, assim como em seu estudo com pessoas com lesão vertero-modular, observa-se entre os participantes deste estudo que cerca de $80 \%$ dos participantes não apresentam indicadores de depressão (Ferreira \& Guerra, 2014).

Estes resultados apontam para necessidade de aprofundar estudos sobre as capacidades psicológicas de adaptação humana frente a situações gravemente estressoras. Apoiados em modelos da Psicologia Positiva é crescente o número de estudos que salientam a forte capacidade das pessoas de alcançarem níveis elevados de adaptação e satisfação subjetiva mesmo frente a perdas importantes e situações de grande incerteza.

Os estudos sobre vulnerabilidade psicológica frente a doenças crônicas são fundamentais para lançar luz sobre as necessidades de avaliação e intervenção precoce para saúde mental desta população. No entanto, faz-se muito importante observar por outro lado quais são os fatores psicossociais que podem mediar o ajustamento psicológico observado em uma população submetida a estressores crônicos. Acessar e depurar essas variáveis poderá permitir grande colaboração a programas de prevenção e desenvolvimento de habilidades de vida e também aperfeiçoar as intervenções de saúde mental atualmente indicadas para pessoas acometidas por doenças crônicas (Scheffold, Mehnert, Müller, Koch, Härter \& Vehling, 2014; Reilly, Lee, Laux, \& Robitaille, 2017).

Aspectos protetivos do desenvolvimento humano, como sentido de vida merece maior investigação e realce, sendo ainda pouco explorados. Apesar da crescente preocupação na compreensão e na avaliação do coping princi- 
palmente em situações estressantes - como o diagnóstico de neoplasia maligna, internação e tratamento -, ainda é evidente a necessidade de mais estudos sobre a temática.

\section{Conclusão}

$\mathrm{O}$ presente estudo demonstrou que a busca de sentido está associada a um menor índice de depressão, ansiedade e desamparo. Portanto é um possível indicador de proteção à saúde mental dos pacientes em tratamento de câncer. Diante de um tratamento altamente agressivo como o câncer o paciente se vê obrigado a rever sua vida e a buscar novos significados.

\section{Referências}

Aquino, T.A.A., Veloso, V. G., Aguiar, A. A., Serafim, T. D. B., Pontes, A. M., Pereira, G. A. \& Fernandes, G. A. (2015). Questionário de Sentido de Vida: Evidências de sua Validade Fatorial e Consistência Interna. Psicol. cienc. prof. vol.35 no.1 Brasília Jan./Mar. 2015. http://dx.doi.org/10.1590/19823703001332012.

Barbosa, M. R., \& Santos, F. U. D. (2012). Fontes estressoras no paciente com diagnóstico de neoplasia mamária maligna. Revista Brasileira de Terapias Cognitivas, 8(1), 10-18.

Botega, N. J., Bio, M. R., Zomignani, M. A., Garcia Jr, C., \& Pereira, W. A. B. (1995). Transtorno de humor em enfermaria de clínica médica e validação de escala de medida (HAD) de ansiedade e depressão. Revista de Saúde Pública, 29(5), 355-363. DOI: 10.1590/S0034-89101995000500004.

Boscaglia, N., \& M Clarke, D. (2007). Sense of coherence as a protective factor for demoralization in women with a recent diagnosis of gynecological cancer. Psycho'Oncology, 16(3), 189-195.

Bronk, K. C. (2014). Purpose in Life: A Critical Component of an Optimal Youth Development. New York, US: Springer.

Casellas- Grau, A., Ochoa, C. \& Ruini, C. (2017). Psychological and clinical correlates of posttraumatic growth in cancer: A systematic and critical review. Psychooncology. Dec;26(12):2007-2018. doi: 10.1002/pon.4426. Epub 2017 May 24.

Casale, M., Wild, L., Cluver, L., \& Kuo, C. (2015). Social support as a protective factor for depression among women caring for children in HIV-endemic South Africa. Journal of behavioral medicine, 38(1), 17-27.

Cho, E., Lee, D., Lee, J. Y., Bae, B. H., \& Jeong, S. M. (2014). Meaning in life and school adjustment: testing the mediating effects of problem-focused coping and self-acceptance. Procedia- Social and Behavioral Sciences, 114, 77-781.

Damásio, B. F., Silva, J. P, Melo, S. A., \& Aquino, T. A. A. (2010). A saúde dos professores por uma expectativa existencial. Em: T. A. A. de Aquino. B. F. Damásio, J. P. da Silva (orgs.). Logoterapia e Educação: fundamentos e prática. pp. 139-153. São Paulo: Paulus

Datu, J. A. (2015). The synergistic interplay between positive emotions and maximization enhances meaning in life: a study in a collectivist context. Current Psychology. doi 10.1007/s12144-015-9314-1.

Dezutter, J., Casalin, S., Wachholtz, A., Luyckx, K., Hekking, J., \& Vandewiele, W. (2013). Meaning in life: An important factor for the psychological well-being of chronically ill patients. Rehabilitation psychology, 58(4), 334.

Dobríková, P., Pčolková, D., AlTurabi, L. K., \& West Jr, D. J. (2015). The Effect of Social Support and Meaning of Life on the Quality-of-Life Care for Terminally Ill Patients. American Journal of Hospice and Palliative Medicine ${ }^{\circledR}, 32(7)$, 767-771.

Dua, R., Das, A., Kumar, A., Kumar, S., Mishra, M., \& Sharma, K. (2018). Association of comorbid anxiety and depression with chronic obstructive pulmonary disease. Lung India, 35(1), 31.

Eakman, A. M., \& Eklund, M. (2012). The relative impact of personality traits, meaningful occupation and occupational value on meaning in life and life satisfaction. Journal of occupational Science, 19(2), 165-177.http: // dx. doi. Org. /10.1080/ 14427591.2012.671762.
Fabry, J. (1984) A busca do significado. São Paulo: ECE.

Frankl, V. E. (1978). Fundamentos antropológicos da psicoterapia. Rio de Janeiro: Zahar.

Frankl, V. E. (1984). Em busca de sentido. Petrópolis: Vozes.

Frankl, V. E. (1991). A psicoterapia na prática. São Paulo: Papirus.

Ferreira, M. \& Guerra, M. (2014). Adaptação à lesão vertebro-medular Psicologia, Saúde \& Doenças,15(2), 380-395. http://dx.doi. org/10.15309/14psd150205

Gandini, R. C., Martins, M. C. F. \& Pedrosa, E. C. M. (2008). Psicologia em Estudo, Maringá, v. 13, n. 1, p. 169-177

Gomes, J. C. V. (1987). Logoterapia. Apsicoterapia existencial humanista de Viktor Emil Frankl. Rio de Janeiro: Vozes. (Coleção Logoterapia).

Griep, R. H, Chor, D., Faerstein, E., Werneck. G. L \& Lopes, C. S. (2005). Validade de constructo de escala de apoio social do Medical Outcomes Study adaptada para o português no Estudo Pró-Saúde. Cad. Saúde Pública vol.21 no.3 Rio de Janeiro May/June 2005. http://dx.doi.org/10.1590/ S0102-311X2005000300004

Herth K. Development and refinement of an instrument to measure hope. Sch Inq Nurs Pract. 1991;5(1):39-51.

Kashdan, T. B. \& Kane, J. Q. (2011). Posttraumatic distress and the presence of posttraumatic growth and meaning in life: Experiential avoidance as a moderator. Pers Individ Dif. Jan 1;50(1):84-89.

Kim, Y. J., \& Lee, K. J. (2010). Relationship of Social Support and Meaning of Life to Suicidal Thoughts in Cancer Patients. Journal of Korean Academy of Nursing, 40(4).

Letborgh, C., Aranda, S., \& Kissane, D. (2008). Meaning in adjustment to cancer: A model of care. Palliative \& supportive care, 6(1), 61-70.

Linard, A. G., Silva, F. A. D., \& Silva, R. M. (2002). Mulheres submetidas a tratamento para câncer de colo uterino - percepção de como enfrentam a realidade. Revista Brasileira de Cancerologia, 48(4): 493-498 493. Disponível em: http://www.inca.gov.br/rbc/n_48/v04/pdf/artigo1.pdf. Acesso em: 09/04/09.

Ortiz, E. M. \& Morales, C. C. (2013). Percepción de sentido de vida en universitarios colombianos. Pensamiento Psicológico, 11(1), 71-82.

Park, J., \& Baumeister, R. F. (2017). Meaning in life and adjustment to daily stressors. The Journal of Positive Psychology, 12(4), 333-341.

Pratti, G., \& Pietrantoni, L. (2009). Optimism, social support, and coping strategies as factors contributing to posttraumatic growth: A meta-analysis. Journal of loss and trauma, 14(5), 364-388.

Reilly, R. C., Lee, V., Laux, K., \& Robitaille, A. (2017). Creating doorways: finding existential meaning and growth through the creative arts in the face of life-threatening illness.

Scheffold, K., Mehnert, A., Müller, V., Koch, U. Härter, M. \& Vehling, S. (2014). Sources of meaning in cancer patients - influences on global meaning, anxiety and depression in a longitudinal study. Volume 23, July, Pages: 472-480. https://doi.org/10.1111/ecc.12152

Schroevers, M. J., Helgeson, V. S., Sanderman, R., \& Ranchor, A. V. (2010). Type of social support matters for prediction of posttraumatic growth among cancer survivors. Psycho'Oncology, 19(1), 46-53.

Sebben, R. (2007). Psiconcologia: o paciente e a família frente ao diagnóstico e tratamento do câncer. Universidade Vale do Itajaí Centro de educação de ciências da saúde. Itajaí, SC.

Sherman, A. C. \& Simonton, S (2011). Effects of personal meaning among patients in primary and specialized care: associations with psychosocial and physical outcomes. Psychol Health. 2012;27(4):475-90. doi: 10.1080/08870446.2011.592983. Epub 2011 Jul 4.

Sharafkhaneh, A., Yohannes, A. M., Hanania, N. A., \& Kunik, M. E. (Eds.). (2017). Depression and Anxiety in Patients with Chronic Respiratory Diseases. Springer.

Snoerck, F. J., Bremmer, M. A., \& Hermanns, N. (2015). Constructs of depression and distress in diabetes: time for an appraisal. The Lancet Diabetes \& Endocrinology, 3(6), 450-460.

Tedeschi, R. G. \& Calhoun, L. G. (1996). The posttraumatic growth inventory: Measuring the positive legacy of trauma. Journal of traumatic Stress. Volume 9, Issue 3. Pages: 455-471 https://doi.org/10.1002/jts.2490090305

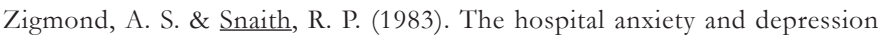
scale. Acta Psychiatr Scand. 1983 Jun;67(6):361-70 
Yalçin, I., \& Malkoç, A. (2014). The relationship between meaning in life and subjective well-being: forgiveness and hope as mediator. Journal of Happiness Studies. doi 10.1007/s10902-014-9540-5.

Kleftaras, G. \& Psarra, E. (2012). Meaning in life, psychological well-being and depressive symptomatology: A comparative study. Psychology, 3(04), 337.

Zeligman, M., Varney, M., Grad, R. I., \& Huffstead, M. (2018). Posttraumatic Growth in Individuals With Chronic Illness: The Role of Social Support and Meaning Making. Journal of Counseling \& Development, 96(1), 53-63.

Zhou, X., Wu, X., \& Zhen, R. (2018). Self-esteem and hope mediate the relations between social support and post-traumatic stress disorder and growth in adolescents following the Ya'an earthquake. Anxiety, Stress, \& Coping, 31(1), 32-45

Yoong, R. K., Mooppil, N., Khoo, E. Y., Newman, S. P., Lee, V. Y., Kang, A. W., \& Griva, K. (2017). Prevalence and determinants of anxiety and depression in end stage renal disease (ESRD). A comparison between
ESRD patients with and without coexisting diabetes mellitus. Journal of psychosomatic research, 94, 68-72.

Vickberg, S. M. J., Bovbjerg, D. H., DuHamel, K. N., Currie, V., \& Redd, W. H. (2000). Intrusive thoughts and psychological distress among breast cancer survivors: Global meaning as a possible protective factor. Behavioral medicine, 25(4), 152-160.

Vanderlan, J., Kurtz, E., Sladky, S., Asvat, Y., \& Deshields, T. (2016). Relationships among Resilience, Depression, Anxiety, and Quality of Life in Cancer Patients Referred to a Psycho-Oncology Service. Psycho-oncology, 25, 65-66.

Submetido em: 27-2-2018

Aceito em: 11-1-2019 